\title{
Herpesviruses: epidemiology, pathogenesis, and interventions
}

\author{
Ke $\operatorname{Lan}^{1 凶}$, Min-Hua Luo ${ }^{2 \bowtie}$
}

\section{State Key Laboratory of Virology, College of Life Sciences, Wuhan University, Wuhan 430072, China \\ 2. State Key Laboratory of Virology, CAS Center for Excellence in Brain Science and Intelligence \\ Technology (CEBSIT), Wuhan Institute of Virology, Chinese Academy of Sciences, Wuhan 430071, China}

This special issue of the journal is dedicated to the recent research progress on human herpesviruses (HHVs). Human herpesviruses are distributed worldwide, and more than $90 \%$ of adults are infected by one or multiple HHVs. The HHV family contains three sub-families: the alpha sub-family [herpes simplex virus 1 (HSV-1), HSV-2, and varicella-zoster virus (VZV)], beta sub-family [human cytomegalovirus (HCMV), HHV6, and HHV7)], and gamma sub-family [Epstein-Barr virus (EBV) and Kaposi's sarcoma-associated herpesvirus (KSHV)]. All the viruses typically establish latent infection in host, and undergo lytic reactivation in certain pathophysiological conditions. Figure 1 illustrates a typical herpesvirus virion, which is a spherical particle with $150-200 \mathrm{~nm}$ in diameter, containing a $\mathrm{T}=16$ icosahedral capsid and glycoprotein spikes protruding out. In this issue, we collectively present ten articles focusing on the epidemiology, pathogenesis, and interventions of HSV-1, VZV, HCMV, EBV and KSHV respectively, and these high-quality review and research articles are contributed by experts on those specific viruses.

The first part deals with the prevalence of Kaposi's sarcoma-associated herpesvirus (KSHV). Firstly discovered in 1994, KSHV is the etiologic agent associated with Kaposi's sarcoma (KS), primary effusion lymphoma (PEL), and multicentric Castleman disease (MCD), all of which arise preferentially in the immunocompromised people. KSHV prevalence varies among geographical regions and populations. Zeng and colleagues report an investigation on the KSHV prevalence in Uygur and Han populations from the Urumqi and Kashgar regions of Xinjiang, China. They find the potential correlation between KSHV prevalence and different ethnicities in endemic areas of Xinjiang, and several environmental factors including population migration may affect KSHV infection rates or KS incidence (Zheng et al., 2017). Meanwhile, Zhang and colleagues report a comprehensive meta-analysis on the epidemiological features of
KSHV among intravenous drug users (IDUs) worldwide, and find IDUs are at higher risk of the KSHV infection globally, when compared with the general population, regardless of geographical region or HIV-infection status (Fang et al., 2017).

The second part of this issue focuses on the pathogenesis and virus-host interactions during herpesvirus infections, and includes several review and research articles. A review article by Qin and colleges summarizes the latest progresses on the role of lipid biosynthesis and metabolism in the pathogenesis of KSHV (Dai et al., 2017). As growing studies have shown that herpesviruses can hijack extracellular vesicles to ensure their survival and persistence, Lu et al. provide an updated review on the emerging functions and effects of extracellular vesicles that herpesviruses utilize for virus infection-associated intercellular communication and microenvironment alteration, which gives us a better understanding about the pathogenesis of herpesviruses (Liu L et al., 2017). As

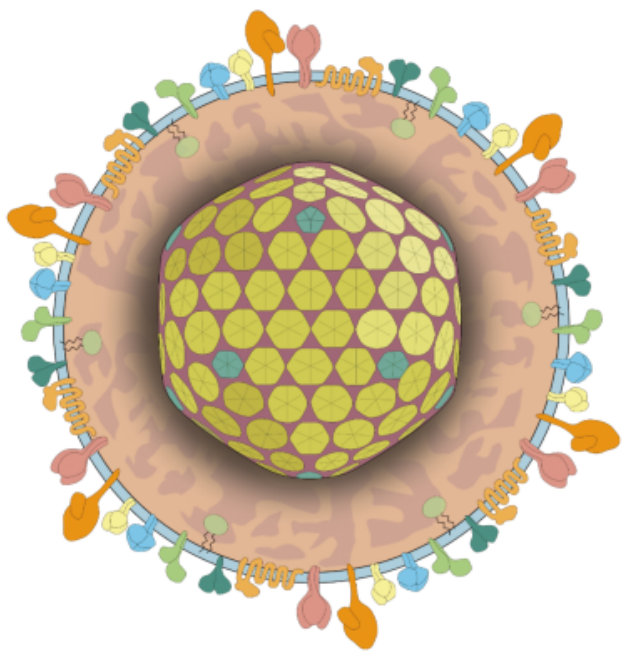

Figure 1. Illustration of a typical herpesvirus virion, modified from the ViralZone. (http://viralzone.expasy.org/5816) 
virus can also make changes to the host intracellular signaling to facilitate its own propagation and survival, Cai and colleagues discuss the recent progresses on molecular properties and regulatory modes of cellular and viral proteins phosphorylation influenced by two human oncogenic gamma-herpesviruses (EBV and KSHV), and highlight the potential therapeutic targets and strategies against their related cancers (Wang Y et al., 2017).

Rasmussen's encephalitis (RE) is a rare pediatric neurological disorder, but its etiology remains unclear. An and colleagues analyze the expression of Epstein-Barr virus (EBV) antigens as well as of Toll-like receptor 3 (TLR3), TLR9, and downstream adapter TIR-domaincontaining adapter-inducing interferon- $\beta$ (TRIF) in the brain tissues of RE patients, and propose that the elevated expression of EBV and TLRs may be involved in RE occurrence through the activation of downstream molecules (Wang X et al., 2017). Varicella-zoster virus (VZV) is a neurotropic alphaherpesvirus that causes chickenpox and shingles, while ORF7 is an important virulence determinant of VZV in both human skin and nerve tissues. Cheng and colleagues describe the study on the properties of ORF53, a partner protein of ORF7. The trans-Golgi network localization of the complex is identified, which may be implicated in VZV pathogenesis (Wang W et al., 2017). The group led by Professor Ruan focuses on the HCMV infection mechanism. They discover Geminin, a DNA replication inhibitor, is a direct target of HCMV-miR-US5-1. Through this interaction, HCMV-miR-US5-1 may affect viral replication and host cellular environment by regulating expression kinetics of Geminin during HCMV infection (Jiang et al., 2017).

The final part of the special is dedicated to the interventions against viral infections. Currently, the HSV-1 vaccine development is still a global challenge. The $\mathrm{Li}$ group reports an attenuated HSV-1 virus generated from a serial virus mutants. Attenuation is tested in both acute and latent infections in mice, and is found to be a potential candidate for HSV-1 vaccine development (Xu et al., 2017). HCMV is the most common viral cause for congenital infection worldwide, and is the leading nongenetic cause of sensorineural hearing loss in children. The review by Liu G et al. highlights the recent progress in diagnostic techniques that could potentially be used for the detection of HCMV infection in neonates and its direct implications in public health settings for diagnosing congenital HCMV infection (Liu G et al., 2017).

This special issue would also acknowledge to the Chinese Herpesvirus Workshop (CHW). The CHW was established spontaneously by several herpesvirus researchers in China including Drs. Ke Lan, Hongyu Deng, Yan Yuan, Chun Lu, Minhua Luo, Qiang Ruan et al. in 2013. Thereafter CHW organizes symposium annually at different cities across China, and the attendees have grown from several research groups to a network of more than 60 institutions. The CHW aims to foster communication, idea exchange and share materials among scientists not only in China but all over the world. The CHW symposiums discuss topics on both basic research and clinical aspects, and the 2018 workshop (the sixth) will be held in Shanghai, co-organized by Drs. Qiliang Cai, Zhikang Qian, Qiming Liang and Tiejun Zhang.

It is hoped that readers could find useful information from this issue, and future studies would further advance our knowledge on herpesviruses.

\section{FOOTNOTES}

We would like to thank all authors for their excellent contributions to this special issue, and the editorial office of Virologica Sinica for their great support. This work was supported by grants from the Natural Science Foundation for Distinguished Young Scholars (81425017), the Ministry of Science and Technology of China (2016YFA0502100), the Key Project of the Natural Science Foundation of China (81230037), and the National Institutes of Health (1R01AI116442) to KL, and the Natural Science Foundation of China (81620108021) to MHL.

$\triangle$ Correspondence:

Ke Lan, Phone: +86-27-68754592, Fax: +86-27-68754592,

Email: klan@whu.edu.cn

ORCID: 0000-0002-0384-8598

Min-Hua Luo, Phone: +86-27-87197600,

Fax: +86-27-87197600,

Email: luomh@wh.iov.cn

ORCID: 0000-0001-9352-0643

Published online: 31 October 2017

\section{REFERENCES}

Dai L, Lin Z, Jiang W, Flemington EK, Qin Z. 2017. Virol Sin, 32: 369-375.

Fang Q, Liu Z, Zhang Z, Zeng Y, Zhang T. 2017. Virol Sin, 32: 415-422.

Jiang S, Huang Y, Qi Y, He R, Liu Z, Ma Y, Guo X, Shao Y, Ruan Q. 2017. Virol Sin, 32: 431-439.

Liu G, Hai R, Liu F. 2017. Virol Sin, 32: 376-386.

Liu L, Zhou Q, Xie Y, Zuo L, Zhu F, Lu J. 2017. Virol Sin, 32: 349-356.

Wang W, Fu W, Pan D, Cai L, Ye J, Liu J, Liu C, Que Y, Xia N, Zhu H, Cheng T. 2017. Virol Sin, 32: 387-395.

Wang X, Wang Y, Liu D, Wang P, Fan D, Guan Y, Li T, Luan G, An J. 2017. Virol Sin, 32: 423-430.

Wang Y, Banerjee S, Ding L, Cai C, Wei F, Cai Q. 2017. Virol Sin, 32: $357-368$.

Xu X, Guo Y, Fan S, Cui P, Feng M, Wang L, Zhang Y, Liu Y, Zhang X, Li Q. 2017.Virol Sin, 32: 404-414.

Zheng J, Yang Y, Cui M, Shu ZJ, Han LL, Liu ZQ, Wood C, Zhang T, Zeng Y. 2017. Virol Sin, 32: 396-403. 